\section{Potential impact of primate-specific SVA retrotransposons during the evolution of human cognitive function}

Olga Vasieva,1 Sultan Cetiner, ${ }^{1}$ Abigail Savage, ${ }^{2}$ Gerald G. Schumann, ${ }^{3}$ Vivien J. Bubb,2 John P. Quinn²

1 Institute of Integrative Biology, University of Liverpool, UK;

2Department of Molecular and Clinical Pharmacology, Institute of Translational Medicine, University of Liverpool, UK; 3Division of Medical Biotechnology, Paul-Ehrlich-Institut, Langen, Germany

\section{Abstract}

The SVA family of hominid-specific non-LTR retrotransposon comprises the youngest group of transposable elements in the human genome. The propagation of the most ancient SVA subfamily took place about 13.5 million years ago, and the youngest SVA subfamily appeared in the human genome after the human/chimpanzee divergence. Functional analysis of genes associated with SVA insertions demonstrated their link to multiple ontological categories, with one of the major categories being attributed to brain function. Further analysis of this subset demonstrated that SVA elements expanded their presence in the human genome at different stages of hominoid evolution and were associated with progressively evolving behavioral features that indicate a potential impact of SVA propagation on the cognitive ability of a modern human. Our analysis suggests a potential role of SVAs in the evolution of human central nervous system and especially in the emergence of functional trends relevant to social and parental behavior. Coevolution of behavioral features and reproductive functions are suggested by our analysis and discussed.

\section{Introduction}

Genetic studies have been successful at determining the changes that alter pathways involved in evolution. Transposable elements (TEs), despite long being thought of as junk DNA, have impacted the human genome during its evolution through a variety of mechanisms causing structural varia- tions including insertional mutagenesis, generation of deletions at the insertion site, $3^{\prime}$ or 5' transduction events, non-allelic homologous recombination and exonisation. ${ }^{1-4}$ More than 10,000 TE insertions occurred in the human genome since human-chimpanzee divergence which were suggested to have implications on human evolution and especially its reproductive, cognitive and immune functions that diverged strongly during a relative short time period.4,5 The large number of such TEs in the genome makes an analysis of their specific contribution to evolution very difficult, and previous studies highlighted the potential role of mainly self-propagating (L1, ERV/ETR) and Alu elements. ${ }^{4}$ We have focused on the functions of genes in proximity to the integration sites of the less studied hominoid-specific composite retrotransposons termed SINE-VNTR-Alu (SVA) elements. ${ }^{6}$ SVA elements represent the youngest non-LTR retrotransposon family in humans and the human genome harbors approximately 2700 SVAs. ${ }^{7-9}$ We and others have demonstrated that SVA elements have properties of transcriptional regulators of gene expression both in vivo and in vitro, $8,10,11$ and that a significant number of SVA elements are located within $10 \mathrm{~kb}$ of the major transcriptional start site of many genes. ${ }^{8}$ Therefore, SVA insertions established in the hominoid lineage could be responsible for altering the transcriptome in a developmental, tissue-specific or stimulus inducible manner, as was already suggested earlier for Alu elements and demonstrated for LTR sequences of endogenous retroviruses. 4

SVA elements are categorized into subfamilies A to F and F1,11 and their age was estimated at 13.56 million years (Myrs) for the oldest subfamily (A) and 3.18 Myrs for the youngest subfamily (F). Subfamily D is by far the largest and encompasses $44 \%$ of all SVAs in the human genome. The subfamily termed F1 represents the smallest group covering only $3 \%$ of all SVA elements. Subfamilies E, F and F1 are human-specific and correspond to the period since the human chimpanzee divergence that occurred $\sim 6$ million years ago. 5 The implications of SVA insertions for the evolution of other hominoid lineages is quite obvious, because, for instance, the family of gibbon-specific LAVA retrotransposons, derived from an SVA A element, has been implicated in the molecular mechanism underpinning genome plasticity of the gibbon lineage. 12 We have previously examined in detail the SVA insertions found upstream of the genes PARK7 and FUS, which are genes implicated in neurodegeneration, and demonstrated that the SVAs associated with these genes have tran-
Correspondence: Olga Vasieva, Institute of Integrative Biology, Department of Comparative Genomics, University of Liverpool, Liverpool, L69 7ZB, UK.

Tel.: +44.151.795.4456 - Fax: +44.151.795.4406. E-mail: info@ingenets.com

Key words: SVA, retrotransposons, hominoid, brain, evolution.

Contributions: OV conceived of the study, designed and performed the systems analysis, and drafted the manuscript, SC performed the computational analysis and took part in drafting of the manuscript, AS has performed the retrieval of SVA-associated gene sets and helped to draft the manuscript, GGS participated in data analysis and helped to draft the manuscript, VJB participated in retrieval of associated gene sets and helped to draft the manuscript, JQ conceived of the study, coordinated SVA-associated gene set retrieval and took part in drafting of the manuscript.

Conflict of interest: the authors declare no potential conflict of interest.

Received for publication: 21 March 2016. Revision received: 24 October 2016.

Accepted for publication: 24 October 2016

This work is licensed under a Creative Commons Attribution NonCommercial 4.0 License (CC BY-NC 4.0).

(C) Copyright O. Vasieva et al., 2017

Licensee PAGEPress, Italy

Trends in Evolutionary Biology 2017; 6:6514 doi:10.4081/ni.2017.6514

scriptional regulatory properties both in vivo and in vitro. ${ }^{7}$ In this communication, we wanted to further explore the potential of members of the different SVA subfamilies (A-F1) to modulate specific genes involved in CNS function. Here, we demonstrate that SVA insertion and subfamily appearance in evolution correlate with the acquisition of certain behavioral traits.

\section{Materials and Methods}

Generation of the list of genes associated with SVA insertions

Genomic coordinates of all SVA loci in the human genome ( $\mathrm{Hg} 19$ reference genome sequence) were extracted from the UCSC genome browser (http://genome. ucsc.edu/index.html). This included many SVA sequences that were fragmented in the Repeat Masker track; therefore, this list was manually annotated to generate a list of coordinates of complete SVA sequences 
resulting in a total of 2676 elements encompassing all seven SVA subfamilies. ${ }^{8}$ The coordinates of all known genes and their transcripts were extracted from the UCSC genome browser, and Galaxy software (http://galaxyproject.org/) was used to generate coordinates of the $10-\mathrm{kb}$ genomic regions flanking all known transcripts. Finally, the SVA loci were intersected with the three lists of genomic coordinates (all known genes, $10 \mathrm{~kb}$ upstream and $10 \mathrm{~kb}$ downstream of known genes). Duplicates were removed from each list individually. For functional enrichment analysis, the defined SVA coordinates have been used to produce a shorter list of the Genome Reference Consortium Human Build 38 (GRCh38) genes directly mapped via BIomart Martview service (www. biomart.org/biomart/martview/).

\section{Ingenuity pathway analysis}

Ingenuity Pathway Analysis (IPA) software (Ingenuity Systems, Inc.) was used to investigate biological functions associated with the data sets generated from genes associated with an SVA insertion. ${ }^{13}$ The IPA database is composed of comprehensive information on genes and pathway ontologies. The database contains approximately 200 canonical pathways as well as 27 higher-order disease and function categories for Core functional enrichment analysis. Righttailed Fishers Exact statistical tests was used to calculate whether the likelihood of associations between a set of focus genes and a category was due to a random chance. The tool enabled production of graphical networks that illustrated mapped genes and their functional connections.

\section{Meta-analysis of gene expression}

Genevestigator software was used to perform a meta-analysis of gene expression in different tissues. ${ }^{14}$ For each gene/tissue an average level of expression is determined via automated cross-analysis of normalized published Affymetrix microarray data, filtered for statistically significant values and stored in the Genevestigator database. The highest and lowest detected average expression levels define a 100 -unit scale that is used for presentation of each gene/tissue related average expression value.

\section{Results}

Relevance of SVA-associated genes to neural system and cognitive function

The top ranked categories identified by IPA for the data set generated from genes associated with an SVA insertion were Cardiovascular disease and Glucose tolerance. However the next highest ranked were Guidance of axons and Synaptic processes $(\mathrm{P}=1 \mathrm{E}-5$ and $\mathrm{P}=6 \mathrm{E}-5$ correspondingly). The genes classified to the latter categories were associated with a number of canonical pathways (such as Synaptic Long Term Potentiation or Axonal Guidance Signaling) with no specific enrichment in any of those top-ranked pathways (Supplementary Material). To better reveal functional trends for potential SVA impact on human cognitive function, we focused only on genes relevant to CNS categories (Table 1 and Supplementary Material) and these were re-analyzed by IPA (Supplementary Material). The top-ranked significantly enriched categories $(-\log (\mathrm{P}-$ value) $>12$ ) were of morphology and development of forebrain/telencephalon and multiple categories relevant to axon growth and synaptic processes. From the mapped behavioral categories, Learning and Social Behavior were the generally most enriched $(-\log (\mathrm{P}-\mathrm{value})>4.6)$ categories and Nest building behavior, Nursing, Sexual behavior, Sexual receptivity of female organism, Grooming, Emotional behavior, Aggressive behavior, Vocalization and Walking were the most significantly enriched categories in the SVA B and/or SVA D associated sets of genes. Social Behavior was the only significantly enriched category attributed to the gene sets associated with the youngest SVA subfamilies F and F1. Many categories across the sets were mapped by one or two genes. All the defined behavioral categories were presented against the timeline of occurrence of insertions of the different SVA subtypes in the human genome as shown on Figure 1.

A number of behavioral categories show an evolutionary corresponding pattern of connection to SVA insertions. The walking-category-associated group of genes ([AGTPBP1 (ATP/GTP binding protein 1)], [CACNB4 (Calcium channel, voltagedependent, B4 subunit)], [FXN (Frataxin), SCN8A (Sodium channel, voltage gated, type VIII alpha subunit)], [ATG7 (Autophagy related 7 homolog)] contain SVA D or F insertions while the functions of climbing activity-associated genes [KCNJ6 (Potassium inwardly-rectifying channel, J6)] and [HTT (Huntingtin)] are associated with earlier SVA A and B insertions (Supplementary Material). We may speculate that alteration of the functionality of these genes overall could have had an impact on changing from climbing to the walking moving mode over the period of hominoid evolution. Consistent with that the decline in Nest building activity was also speculated to have an enhancing impact on evolution of walking survival skills, and we see this category associated with two SVA B and D types. In that respect a modulation of a limited number of genes in a specific pathway could have a major effect on the overall pathway. Behavioral characteristics encoded by genes containing younger SVA elements are generally of a more executive nature, for instance [CREB1 (cAMP responsive element binding protein 1)] associated with an SVA E insertion, is involved in both vertical and spatial learning and place preference, while [PSEN1 (Presenilin-1)] is associated with an SVA F element and plays a role in social order recognition, memory and social behavior. In this respect, it attracts our

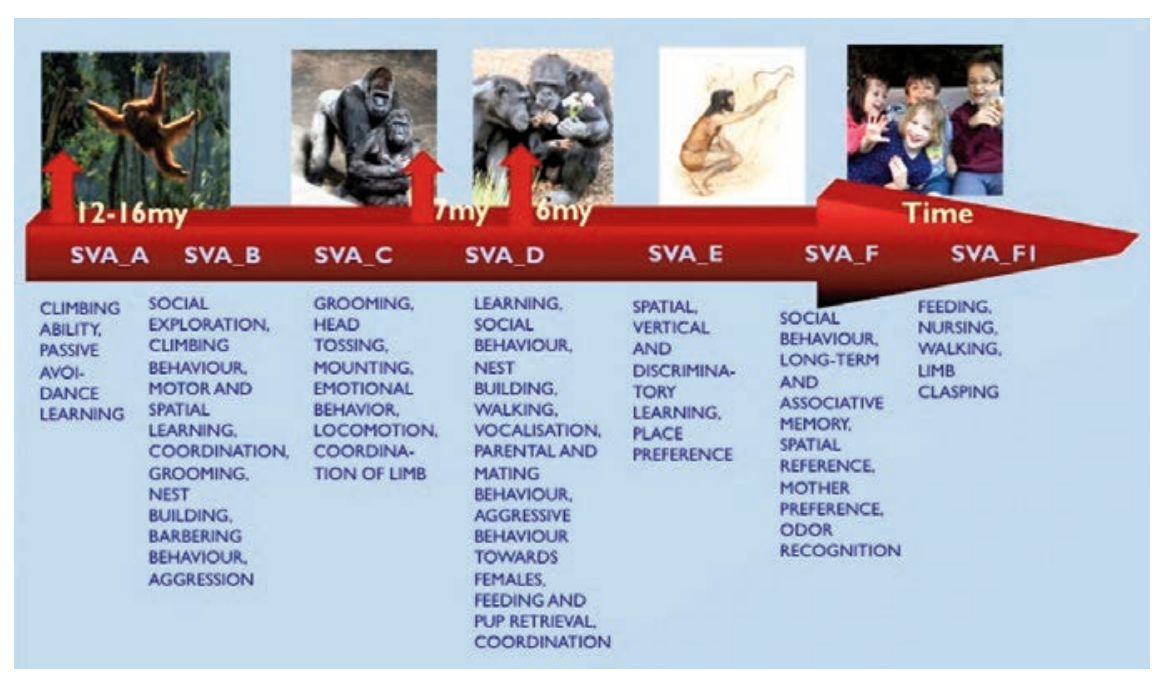

Figure 1. Behavioral categories attributed to genes associated with SVA insertions at different periods during evolution. 
attention that 3 genes with functions relevant to maternity care: Mother preference associated gene - [OMP (Olfactory marker protein)] and metabolic functions associated with Nursing and Feeding categories ([ACOT11 (Acyl-CoA thioesterase)], [MKL1 (Megakaryoblastic leukemia (translocation))]), are also associated with the recent SVA F (Supplementary Material). Interestingly, the number of functions associated with regulation of mating and parental behavior is also affected by SVA D insertions. Among those genes involved are genes encoding regulators of hormonal biosynthesis and response ([CYP19A1 (Aromatase)], [ESR1 (Oestrogen receptor 1)]), metabolism ([LEPR (Leptin receptor)], [ATP8A2 (Phospholipid transporting ATPase 8A2)], [FTO (Alpha-ketoglutaratedependent dioxygenase )]), synaptic function ([SHANK2 (SH3 And Multiple Ankyrin Repeat Domains 2)], [GPHN (Gephyrin)]), and immune response ([IL1RN (IL1 receptor antagonist)], [OCLN (Occludin)]). Several genes found to be associated with SVA insertions are involved in multiple ontological categories, e.g. the genes HTT associated with SVA B, or [BRCA1 (Breast cancer type 1 susceptibility protein)], associated with SVA F, could have been especially significant at particular evolutionary stages whilst having largely pleiotropic effects (Figure 2). It is indicative that genes with different SVA insertions functionally crosstalk. BRCA1, for instance, is not only linked to DNA repair processes but is also involved in interactions with a number of transcription factors, such as ER1, which itself has an SVA insertion (Figure 2). As an extreme example of crosstalk between different SVA subtypes are many cases of distinct subtype insertions in a single gene (Table 1 and Supplementary Material).

\section{Meta-analysis of anatomical rele- vance of SVA-associated behavioral gene expression}

Forebrain and hindbrain were confirmed to be the sites of high expression of the majority of the genes attributed to behavioral phenotypes. High expression levels of the majority of genes associated with all 7 SVA subtypes were also found outside of the CNS which indicates a pleotropic multi-tissue effect of the genes with behavioral phenotypes and suggests their coordinated evolution. Potential positive selection of SVA-associated genes could be attributed, among other factors, to their direct effect on an organism's reproduction. Nearly $80 \%$ of all genes associated with members of the SVA A and B subtypes were highly expressed in placental chorion
(Supplementary Material). Similarly, all genes were expressed in testis at above average levels. A number of the behavioral SVA-associated genes exhibited their highest expression levels in oocytes: [TIMD4 (T-cell immunoglobulin and mucin domain containing 4)], [OXT (Oxytocin/ Neurophysin I)], [KALRN (Kalirin)], [cyp7B1(Cytochrome 7B1)] and already mentioned genes MKL1 and ATG7, which are enriched for SVA F and SVA F1 insertions. Some genes whose expression is known to cause a strong neural phenotype, such as [GABBR2 (GABA B receptor 2)] (SVA A) and CREB1 (SVA E), are also highly expressed in oocytes. Interestingly, germ cells are characterized by increased mobilization of endogenous retrotransposons in general, ${ }^{15}$ and attribution of SVAs to genes expressed in these germ cells might therefore be relevant for the SVA mobilization and propagation in this cell type. Analysis of attribution of the gene set with neuronal and behavioral functions in phenotypic reproductive categories (IPA analysis, Supplementary Material) also highlighted their link to spermatogenesis (in particular, sperm motility [(ADCY10 (Adenylate cyclase 10)]), atrophy of testis and seminiferous tubules (HTT)), infertility in both genders ([STAR (Steroidogenic acute regulatory protein)], [ABCA1 (ATPbinding cassette A1)], [MKKS (McKusickKaufman syndrome)], [NPEPPS
(Puromycin-sensitive aminopeptidase)]), maturation ([LFNG (O-Fucosylpeptide-3Beta-N-acetylglycosyl transferase)]) and fertilisation ([CLIC4 (Chloride intracellular channel 4)]) of oocytes. Several genes, such as [CYP19A1 (Cytochrome 19A1)], [ABL2 (Non-receptor tyrosine kinase)], [SHANK1 (SH3-and-multiple-ankyrin repeat-domains protein 1)] and already mentioned ESR1, CREB1 and NPEPPS also have an association in the IPA knowledgebase with the following behavioral aspects of reproduction: sexual receptivity of female organism, mating behavior, intromission, mounting and pup retrieval.

\section{Discussion}

Retrotransposons have been suggested to be major drivers of genome evolution, both through structural variations such as insertional mutagenesis and modulation of the transcriptome. ${ }^{4,16}$ SVAs can also induce alternative splicing and exon skipping which can result in the generation of alternative transcripts of a gene as documented by disease causing insertions. ${ }^{17-19}$ Thus SVA insertions may generate new interactions between what would otherwise be distinct pathways. 8,20 In this communication, we suggest a more global involvement of genes associated with SVA insertions in

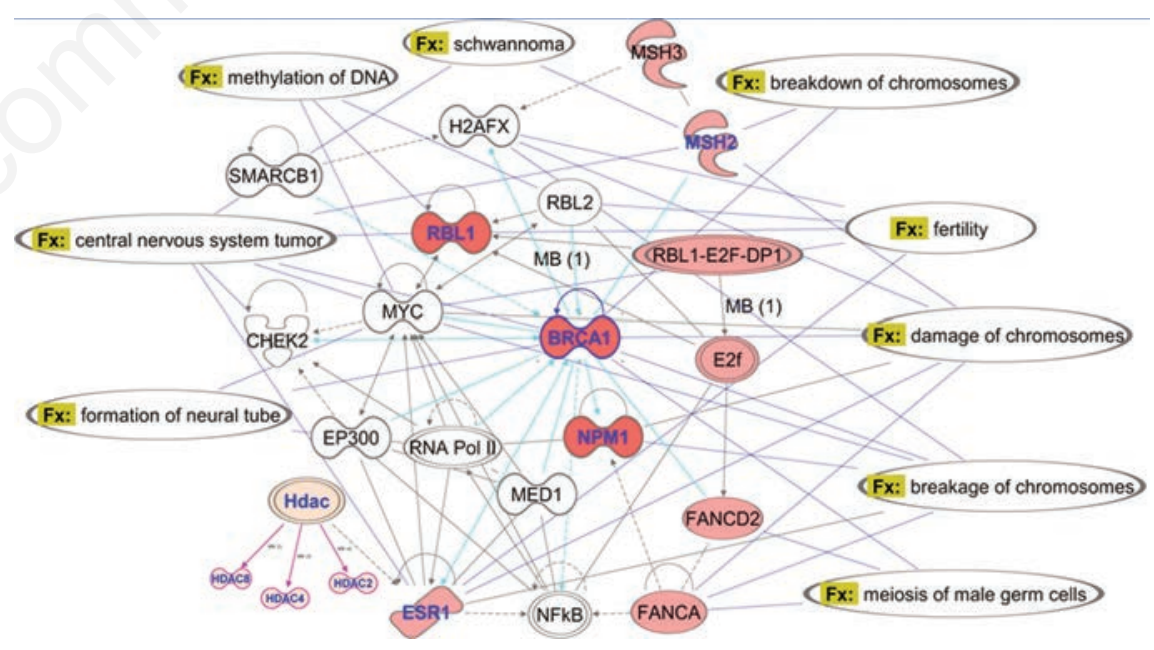

Figure 2. Functional networks reconstructed from SVA-associated genes and classified in neuronal and behavioral categories. Fx-labels correspond to gene-attributed functions and diseases. Solid lines - experimentally validated functional interactions, dashed lines protein binding. The color intensities of blocks correspond to types of intragenic SVA insertions increasing in the order: B, D, F. Blue lettering indicates the genes associated with neuronal and behavioral categories. ACOT11(Acyl-CoA thioesterase 11), [CDK5RAP2 (CDK5 regulatory subunit associated protein 2)], [DIXDC1( DIX domain containing 1)], [E2F (Elongation factor 2)], ESR1 (Estrogen receptor1), [FANCA (Fanconi anemia protein A)], [FANCD2 (Fanconi anemia protein D2)], [HdaC (Histone deacetylase)], MSH2(MutS homolog 2)], [NPM1 (Nucleophosmin)], [POLA1(Polymerase (DNA directed), alpha 1, catalytic subunit)], [RBL1 (Retinoblastoma-like 1)], VPS35 (Vacuolar protein sorting 35 homolog)]. 


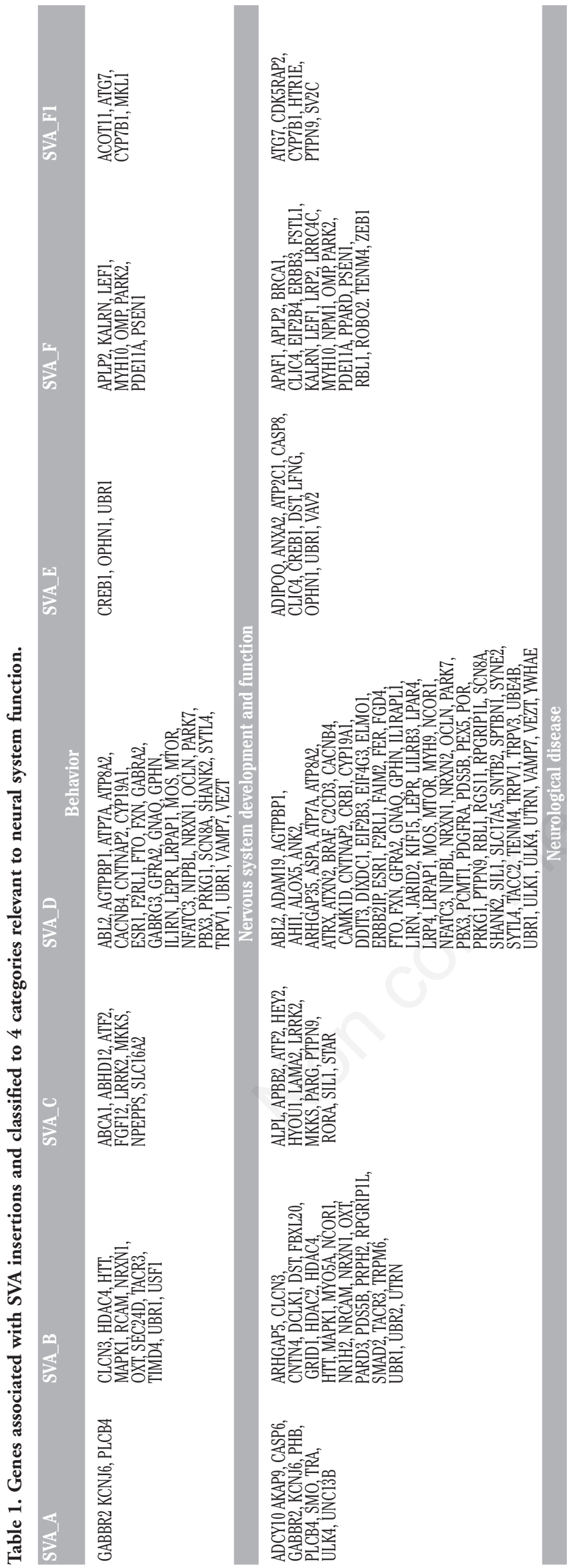

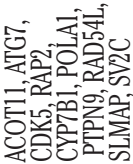

望

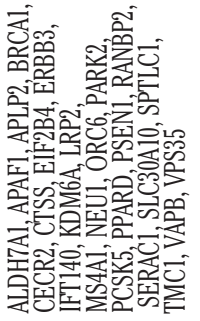

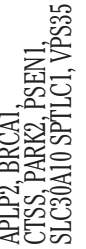

定死

च्रें

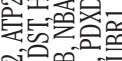

(1)

don

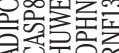

四

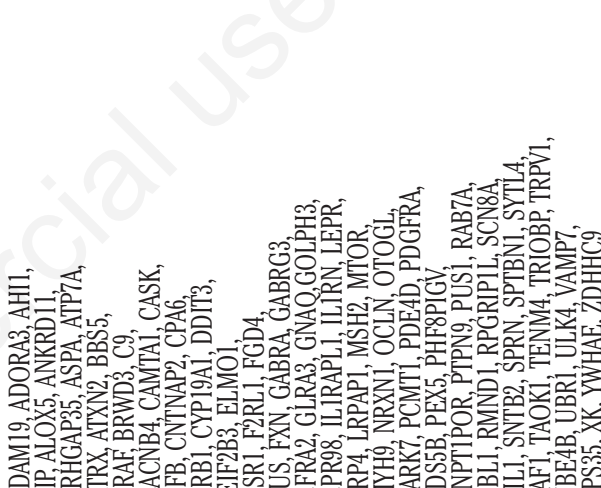

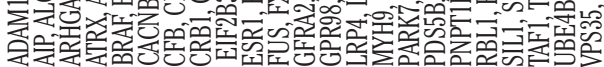
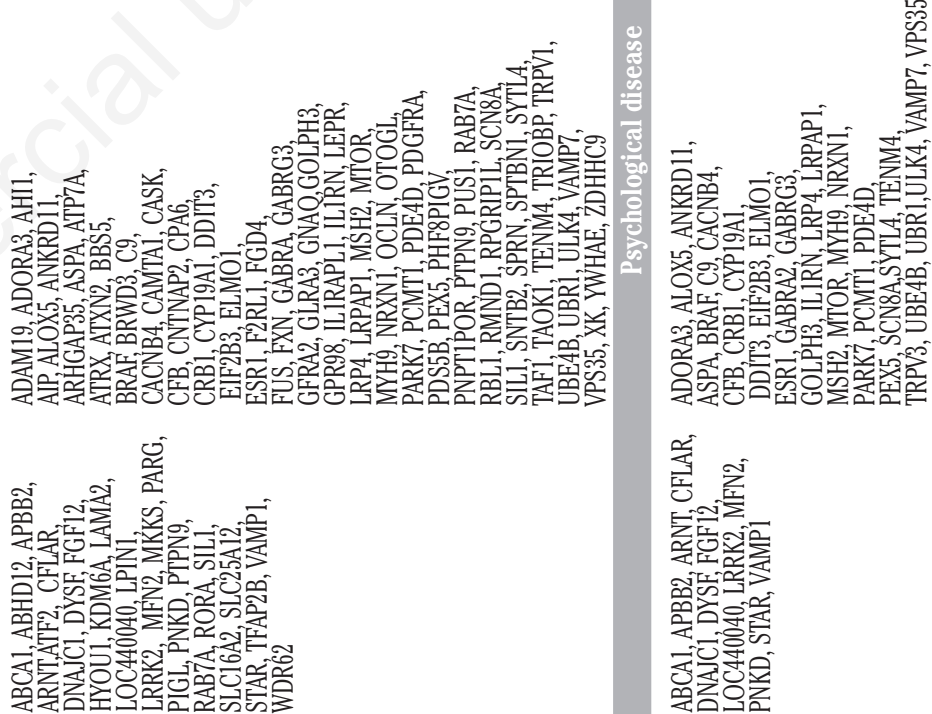

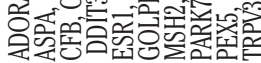

空

运更

空演

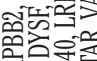

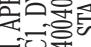

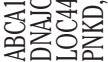

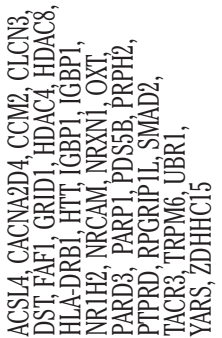

寓

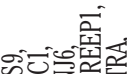

a

(1)

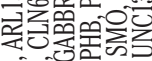

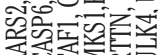

额

峜

[page 4]

[Trends in Evolutionary Biology 2017; 6:6514] 
CNS-relevant pathways. Our data has established that more primitive behavioral characteristics (as climbing, for example) are prominent for the genes associated with the older SVA subtypes A and B and later insertions are more related to younger trends, such as walking. Overall, results revealed a striking correlation between the timing of SVA insertions (Figure 1) and functional significance of these associated genes at particular stages of hominoid evolution. ${ }^{21}$ This may not be surprising but could be consistent with the lineage variation of the phylogenic tree for primates (Figure 1). It attracts our attention, particularly, that a number of behavioral characteristics relevant to maternity care such as Nursing, Feeding, Nest Building, Mother Preference, were populated by SVA-associated genes during the last 6 Myrs of hominoid evolution (Figure 1). Provided these structural changes are functionally significant, they can correspond to a consistent neotenisation of hominoids and development of a familybased social structure. ${ }^{21}$ Another example is Nest Building Behavior that is associated with SVA types B and D and is known to decline with evolution of hominoids due to a switch to ground-sleeping. ${ }^{22}$ This decline was speculated to be also an enhancer of walking survival skills, innovation, procedural memories and gender specification. The associated insertions can suggestively play a suppressive or deleterious role in this behavioral trend. Some of the SVA insertions could also have a gender-specific influence on cognitive functions. The $B R C A 1$ gene, for instance, which is associated with an SVA F insertion and is one of the hubs in SVA integrated functional network (Figure 2), was shown to be in a cross talk with the estrogen receptor ESR1.23,24 $B R C A 1$ is also known to have a strong role in the control of brain size, ${ }^{25,26}$ especially in regions responsible for learning, memory, muscle control and balance. Interestingly, structurally related PGR (progesteronedependent transcription factor) binding sites were shown to be enriched in ancient mammalian TEs linked to evolution of pregnancy, 27 and LTR and Alu elements were also shown to be strongly associated with genes relevant to reproduction. 4

Moderate expression of the majority of behavioral-SVA-associated genes in male germ cells (in contrast to less than $10 \%$ though strongly, expressed in oocyte) could make this cell type a particular source of trans-mobilized SVA elements which are inheritable and passed on to following generations. This hypothesis would be supported by a strong expression of L1 retrotransposons in this niche which are known to provide the protein machinery required for
SVA trans-mobilization. ${ }^{28}$

Behavioral trends caused by SVA insertions in genes expressed in brain could be thus linked to a higher rate of an inheritable SVA trans-mobilization. Thus, being subjected to positive or negative selection at particular stages of hominoid evolution, those behavioral trends could be associated with waves of heritable SVA propagation in the genome. The mechanisms of acceleration and inhibition of propagation of SVAs may be common for all TE, and the wavy nature of this process have been mentioned earlier.4 Genes associated with both neuronal and reproductive phenotypes as well as with TE trans-mobilization properties could be in the first line of the TE-driven evolutionary changes. Interestingly, genes involved in CNS and reproductive functions and DNA repair are associated with different autonomous and non-autonomous TE elements, ${ }^{4}$ that potentially make a cumulative evolutionary impact.

\section{Conclusions}

Our systems analysis suggests an impact of SVA retrotransposition on evolution and performance of human cognitive functions. Data revealed that insertions of members of the older SVA subtypes A-C occurred in genes involved in more primitive characteristics whereas younger SVA D-F1 insertions were present in genes linked to more sophisticated human-specific traits. The presence of SVAs in multiple genes within a network may allow for a concerted response to an environmental stimulus modulated by factors targeting SVA regulatory sequences. Potential positive feedbacks in SVA-associated functional networks and crosstalk between cognitive and reproductive functions would lead to waves of SVA trans-mobilization in the course of hominoid evolution. Considering that genetic polymorphisms in the human population are associated with SVA insertions and that the youngest SVA insertions are documented in human brain and germ line tissues, it can be suggested that SVAs have a modulatory impact on recent human behavioral trends and susceptibilities to psychiatric disorders.

\section{References}

1. Muotri AR, Marchetto MC, Coufal NG, Gage FH. The necessary junk: new functions for transposable elements. Hum Mol Genet 2007;16:R159-67.
2. Hancks DC, Ewing AD, Chen JE, et al. Exon-trapping mediated by the human retrotransposon SVA. Genome Res 2009;19:1983-91.

3. Hancks DC, Kazazian HH, Jr. SVA retrotransposons: evolution and genetic instability. Semin Cancer Biol 2010;20:234-45.

4. Oliver KR, Green WK. Mobile DNA and the TE-Thrust hypothesis: supporting evidence from primates. Mobile DNA 2011,2:8.

5. Mills RE, Bennett EA, Iskow RC, et al. Recently mobilized transposons in the human and chimpanzee genomes. Am J Hum Genet 2006;78:671-9.

6. Ostertag EM, Goodier JL, Zhang Y, Kazazian HH, Jr. SVA elements are non autonomous retrotransposons that cause disease in humans. Am J Hum Genet 2003;73:1444-51.

7. Wang H, Xing J, Grover D, et al. SVA elements: a hominid-specific retroposon family. J Mol Biol 2005;354:9941007.

8. Savage AL, Bubb VJ, Breen G, Quinn JP. Characterisation of the potential function of SVA retrotransposons to modulate gene expression patterns. BMC Evol Biol 2013;13:101.

9. Cordaux R, Batzer MA. The impact of retrotransposons on human genome evolution. Nat Rev Genet 2009;10:691703.

10. Savage AL, Wilm TP, Khursheed K, et al. An evaluation of a SVA retrotransposon in the FUS promoter as a transcriptional regulator and its association to ALS. PLoS ONE 2014;9:e90833.

11. Zabolotneva AA, Bantysh O, Suntsova MV, et al. Transcriptional regulation of human-specific SVAF(1) retrotransposons by cis-regulatory MAST2 sequences. Gene 2012;505:128-36.

12. Carbone L, Harris RA, Gnerre S, et al. Gibbon genome and the fast karyotype evolution of small apes. Nature 2014;513:195-201.

13. Gietzen D. Ingenuity Pathways Analysis (IPA) 2010 [cited 2013]. Available from: h t $\mathrm{t} \mathrm{p}:$ / / w w w . u s c. edu/hsc/nml/assets/bioinfo/IPA/Data $\% 20$ Analysis\%20training\%20Handouts.pdf.

14. Hruz T, Laule O, Szabo G, et al. Genevestigator V3: a reference expression database for the meta-analysis of transcriptomes. Advances in Bioinformatics 2008;2008:420747

15. Levin HL, Moran JV. Dynamic interactions between transposable elements and their hosts. Nat Rev Genet 2011;12:615-27.

16. Thompson P, Macfarlan TS, Lorincz MC. Long terminal repeats: from para- 
sitic elements to building blocks of the transcriptional regulatory repertoire. Mol Cell 2016;62:766-76.

17. Hancks DC, Kazazian HH, Jr. Active human retrotransposons: variation and disease. Curr Opin Genet Dev 2012;22:191-203.

18. Kaer K, Speek M. Retroelements in human disease. Gene 2013;518:231-41.

19. Nakamura Y, Murata M, Takagi Y, et al. SVA retrotransposition in exon 6 of the coagulation factor IX gene causing severe hemophilia B. Int J Hematol 2015;102:134-9.

20. Quinn JP, Bubb VJ. SVA retrotransposons as modulators of gene expression. Mob Genet Elements 2014;4:e32102.

21. Hrdy SB. Evolutionary context of human development: the cooperative breeding model. Carter CS, ed. Cambridge: M.I.T. Press; 2005.
22. Coolidge FL. The effects of the tree-toground sleep transition in the evolution of cognition in early Homo. Before Farming 2006;4:11-8.

23. Gorrini C, Gang BP, Bassi C, et al. Estrogen controls the survival of BRCA1-deficient cells via a PI3KNRF2-regulated pathway. Proc Natl Acad Sci USA 2014;111:4472-7.

24. Kang HJ, Yi YW, Kim HJ, et al. BRCA1 negatively regulates IGF-1 expression through an estrogen-responsive element-like site. Cell Death Dis 2012;3:e336.

25. Pao GM, Zhu Q, Perez-Garcia CG, et al. Role of BRCA1 in brain development. Proc Natl Acad Sci USA 2014;111:E1240-E8.

26. Pulvers JN, Huttner WB. Brcal is required for embryonic development of the mouse cerebral cortex to normal size by preventing apoptosis of early neural progenitors. Development 2009;136:1859-68.

27. Lynch VJ, Nnamani MC, Kapusta A, et al. Ancient transposable elements transformed the uterine regulatory landscape and transcriptome during the evolution of mammalian pregnancy. Cell Rep 2015;10:551-61.

28. Raiz J, Damert A, Chira et al. The nonautonomous retrotransposon SVA is trans-mobilized by the human LINE-1 protein machinery. Nucleic Acids Res 2012;40:1666-83.

29. Zamudio N, Bourchis D. Transposable elements in the mammalian germline: a comfortable niche or a deadly trap quest. Heredity 2010;105:92-104.

30. Han JS, Szak ST, Boeke JD Transcriptional disruption by the L1 retrotransposon and implications for mammalian transcriptomes. Nature 2004;429:268-74. 